\title{
Synthesis of novel lincomycin derivatives and their in vitro antibacterial activities
}

\author{
Eijiro Umemura, Yoshinari Wakiyama, Ko Kumura, Kazutaka Ueda, Satomi Masaki, Takashi Watanabe, \\ Mikio Yamamoto, Yoko Hirai, Hideki Fushimi, Takuji Yoshida and Keiichi Ajito
}

The Journal of Antibiotics (2013) 66, 195-198; doi:10.1038/ja.2012.107; published online 12 December 2012

Keywords: antibiotic; clindamycin; lincomycin; resistant bacteria; S. pneumoniae

\begin{abstract}
INTRODUCTION
Macrolide antibiotics are active against Gram-positive bacteria, especially Streptococcus pneumoniae, and their safety as an oral agent has already been proved. Therefore, macrolide antibiotics are regarded as very important chemotherapeutic agents against bacterial respiratory infections as in the case of $\beta$-lactam antibiotics or new quinolones. Although clarithromycin and azithromycin, which are representatives of widely used macrolides, exhibit enhanced antibacterial activities and characteristic pharmacokinetics compared with those of erythromycin, they are not active enough against resistant bacteria of $S$. pneumoniae with erm gene. Recently these resistant bacteria have widely spread, especially in European and Asian countries, and caused severe social problems. Chemical modifications of erythromycin provided the clinical site with two novel ketolides, telithromycin and cethromycin, which are effective against resistant bacteria of $S$. pneumoniae with erm gene. No oral antibiotic, however, has been launched so far, which is effective against the resistant bacteria of $S$. pneumoniae and does not have any problems in safety or taste.

Lincomycin (LCM) isolated as a secondary metabolite from fermentation broth of Streptomyces lincolnensis was chemically transformed to a useful oral antibiotic, clindamycin (CLDM) (Figure 1), which inhibits bacterial protein synthesis in a similar manner to macrolides. Although telithromycin is still influenced by efflux pumps of resistant S. pneumoniae with mef gene, CLDM is not influenced by efflux pumps. We focused not only on its safety and effectiveness against efflux pumps but its possibility of switch therapy by CLDM analogs, and planned to generate a novel oral antibiotic, which is effective against resistant bacteria of $S$. pneumoniae with erm gene or mef gene by chemical modifications of LCM.
\end{abstract}

\section{BACKGROUND AND MOLECULAR DESIGN}

Regarding chemical modification of LCM, Argoudelis et al. ${ }^{1}$ investigated fundamental SAR analysis focused on the C-6 (N-1 and $\left.\mathrm{C}-4^{\prime}\right)$ and C-7 positions in 1960s and Magerlein et al. ${ }^{2,3}$ generated
U-24,279 A, (Figure 1) which exhibited much stronger in vitro and in vivo activities compared with LCM. On the other hand, Lewis et al. ${ }^{4,5}$ disclosed VIC-105555 (Figure 1) and proved that its in vitro activity against Enterococcus faecalis was much stronger than that of CLDM and its half-life in plasma was greatly improved compared with that of CLDM in a variety of animal species. As a pioneer work, Sztaricskai and Ōmura et al. ${ }^{6}$ reported introduction of a heterocycle via sulfur atom onto the LCM carbon framework ( 1 and 2 shown in Figure 1) with (7R)-configuration, by means of $\mathrm{S}_{\mathrm{N}} 2$ reaction analogous to the synthesis of CLDM. All these derivatives introduced as shown in the above exhibited substantially same antibacterial spectra as those of LCM or CLDM, and there was no description about their antibacterial activities against resistant bacteria of S. pneumoniae with erm gene. Because 3D structural interaction of CLDM-rRNA complex by X-ray single crystallographic analysis ${ }^{7}$ indicated that there were several interactions, mainly a hydrogen bonding, between the peptidyl transferase cavity (A2058Ec, G2520Ec and A2059Ec) and hydroxyl groups at the sugar moiety of CLDM, chemical modification at the sugar part was supposed to be rather difficult for improving activity. As a matter of fact, 2-deoxy-LCM ${ }^{8}$ shows only $1 \%$ activity of LCM, and this result can be explained by lack of the hydrogen bonding between A2058Ec and 2-OH of LCM. We consequently pursued modification at $\mathrm{C}-7$ and then $\mathrm{C}-6\left(\mathrm{~N}-1^{\prime}\right.$ and $\left.\mathrm{C}-4^{\prime}\right)$, focusing on hydrophilic interaction in extra $3 \mathrm{D}$ spaces in the above analysis.

\section{RESULTS AND DISCUSSION}

We paid attention to the fact that stereochemistry at the C-7 position affected antibacterial activity, and synthesized a variety of (7S)-7sulfur-aromatic derivatives of LCM firstly, in application of 2,3,4-tris(trimethylsilyl)- $\mathrm{LCM}^{9}$ as a key intermediate prepared via 2,3,4,7tetrakis-(trimethylsilyl)-LCM. Precise molecular design, practical synthetic procedures and full package of physicochemical properties of each derivative will be reported elsewhere. Although every 


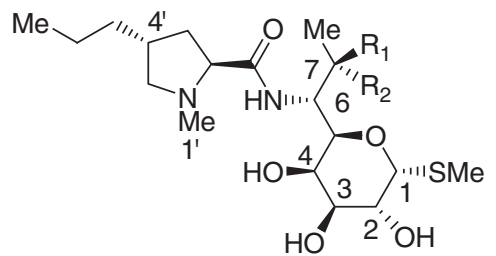

LCM: $\mathrm{R}_{1}=\mathrm{OH} ; \mathrm{R}_{2}=\mathrm{H}$

CLDM: $\mathrm{R}_{1}=\mathrm{H} ; \mathrm{R}_{2}=\mathrm{Cl}$

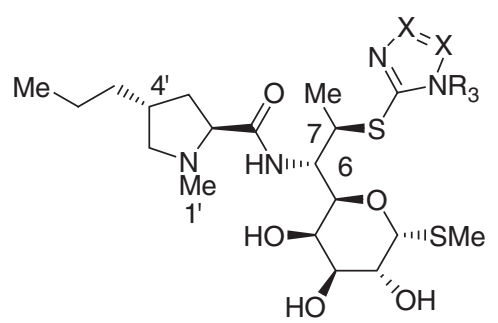

1: $\mathrm{R}_{3}=\mathrm{H} ; \mathrm{X}=\mathrm{CH}$

2: $\mathrm{R}_{3}=\mathrm{Me} ; \mathrm{X}=\mathrm{N}$

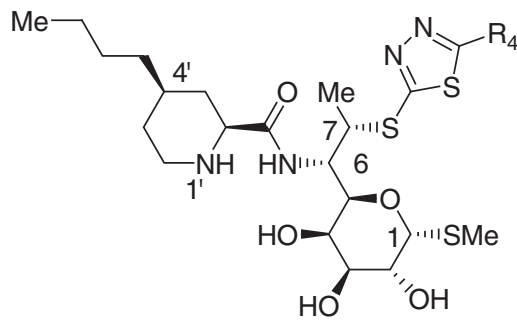

12: $R_{4}=R_{7}$<smiles>CCCCC[C@]1(C)CNC(C(=O)N[C@H]([C@H](C)Cl)[C@H]2O[C@H](C)[C@@H](O)[C@H](O)[C@H]2O)C1</smiles>

U-24,279A

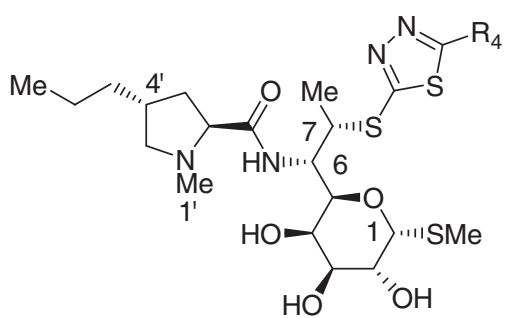

3: $\mathrm{R}_{4}=\mathrm{H}$

4: $\mathrm{R}_{4}=\mathrm{NH}_{2}$

5: $\mathrm{R}_{4}=\mathrm{Ph}$

6: $R_{4}=R_{7}$

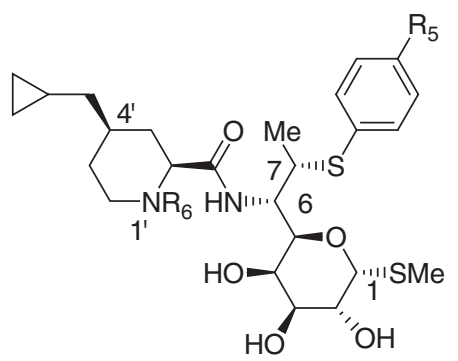

13: $\mathrm{R}_{5}=\mathrm{CH}_{2} \mathrm{CH}_{2} \mathrm{NMe}_{2} ; \mathrm{R}_{6}=\mathrm{Me}$

14: $R_{5}=R_{9} ; R_{6}=M e$

15: $R_{5}=R_{9} ; R_{6}=H$
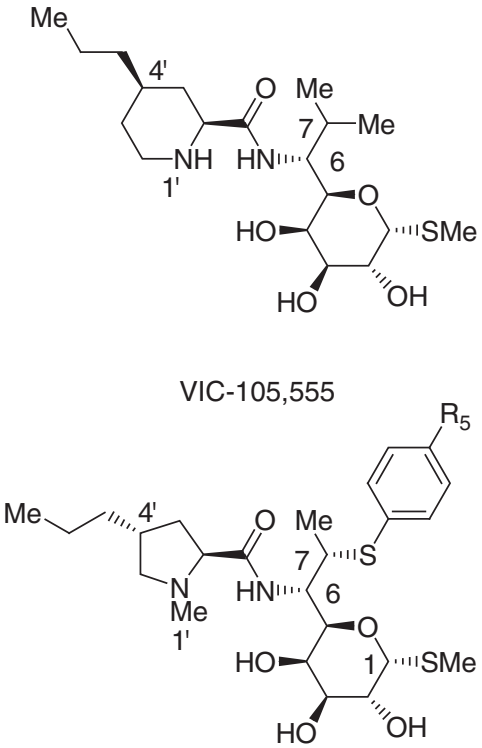

7: $\mathrm{R}_{5}=\mathrm{H} \quad$ 10: $\mathrm{R}_{5}=\mathrm{CH}_{2} \mathrm{CH}_{2} \mathrm{NMe}_{2}$

8: $R_{5}=C^{-O N M e} e_{2}$ 11: $R_{5}=R_{9}$

9: $\mathrm{R}_{5}=\mathrm{COR}_{8}$<smiles>COc1cc(OC)c([N+](=O)[O-])cc1OC</smiles><smiles>[B]N1CCOCC1</smiles><smiles>[R9]c1cncnc1</smiles>

Figure 1 Chemical structures of LCM, CLDM, their reported analogs and novel LCM derivatives.

derivative exhibited almost the same in vitro activities, introducing a substituent to an aromatic ring (1,3,4-thiadiazole or a benzene ring) enhanced antibacterial activity ( 3 to 4 and 7 to 8 ) (Table 1, all antibacterial evaluations were performed as hydrochloride). Representative ${ }^{1} \mathrm{H}$ NMR data and MASS data of each analog are shown in Table 2. Then, we selected compounds 3 and 7 as lead compounds and started systematic synthesis of derivatives of $\mathbf{3}$ and $\mathbf{7}$ through introducing a substituent onto 1,3,4-thiadiazole or a benzene ring.

In the thiadiazole analogs synthesis, 5-phenyl-1,3,4-thiadiazol-2-yl analog (5) exhibited moderate activities against resistant bacteria of S. pneumoniae and Streptococcus pyogenes with erm gene. Then, optimization of 7-sulfur-substituent by the introduction of an additional substituent onto the phenyl group of $\mathbf{5}$ gave key compound 6. In the benzene analogs synthesis, locally optimized compound $\mathbf{9}$ showed stronger activity against the target compared with $\mathbf{8}$, but there seemed to be limitation for further enhancement of in vitro activity. Then, basic functionality or one more aromatic ring (by SAR of compound 5) was introduced to the para-position of the benzene ring of compound 7, and partially optimized compounds $\mathbf{1 0}$ and 11 were prepared. Because compounds 6, 10 and 11 exhibited strong antibacterial activities against clinically important pathogens for respiratory infections (Table 1), we performed final optimization of the whole molecule, especially by handling the proline part.
Ring expansion strategies (from a proline framework to a pipecolic acid framework, see VIC-105,555) have already been reported by Birkenmeyer et al., ${ }^{10,11}$ and we expanded a five-membered ring to sixor seven-membered ring and simultaneously optimized structure of a side chain at the $\mathrm{C}-4^{\prime}$ position. As a conclusion, ${ }^{12-14}$ compounds 12 and $\mathbf{1 3}$ to $\mathbf{1 5}$ were generated as candidates for further biological evaluations. Introduction of a cyclopropylmethyl group to the pipecolic acid moiety in the LCM framework has already been reported by Lewis et al. ${ }^{15,16}$ As shown in Table 1, these derivatives possessed very strong antibacterial activities against not only resistant bacteria of S. pneumoniae with erm or mef gene, but other clinically important pathogens for respiratory infections such as $S$. pyogenes. Among them, compounds 12, 14 and 15 unexpectedly exhibited extremely potent antibacterial activities against resistant $S$. pneumoniae with erm gene even when compared with telithromycin and these derivatives were recognized as the first examples which were effective enough in vitro against the target resistant S. pneumoniae apart from ketolides.

\section{EXPERIMENTAL PROCEDURE}

\section{In vitro antibacterial activity}

Minimum inhibitory concentration was determined by the agar dilution method. Test strains were subjected to seed culture using sensitivity test broth (STB, Nissui Pharmaceutical, Tokyo, Japan) 
Table 1 Antibacterial activities of novel LCM derivatives against $S$. pneumoniae, S. pyogenes and $H$. influenzae. Minimum inhibitory concentration $\left(\mu \mathrm{gml}^{-1}\right)^{\mathrm{a}}$

\begin{tabular}{|c|c|c|c|c|c|c|c|c|c|c|c|c|c|c|c|c|c|c|}
\hline No. & & Characteristics & $L C M$ & $C L D M$ & 3 & 4 & 5 & 6 & 7 & 8 & 9 & 10 & 11 & 12 & 13 & 14 & 15 & TEL \\
\hline 1 & $\begin{array}{l}\text { Streptococcus } \\
\text { pneumoniae DP1 } \\
\text { Type I }\end{array}$ & Susceptible & 1 & 0.13 & 0.13 & 0.03 & 0.06 & 0.015 & 0.25 & 0.03 & 0.06 & $\leqslant 0.008$ & $\leqslant 0.008$ & 0.03 & $\leqslant 0.008$ & $\leqslant 0.008$ & $\leqslant 0.008$ & $\leqslant 0.008$ \\
\hline 2 & $\begin{array}{l}\text { S. pneumoniae } \\
\text { No. } 2\end{array}$ & Susceptible & 1 & 0.13 & 0.25 & 0.03 & 0.13 & 0.015 & 0.25 & 0.03 & 0.06 & 0.015 & $\leqslant 0.008$ & 0.015 & $\leqslant 0.008$ & $\leqslant 0.008$ & $\leqslant 0.008$ & $\leqslant 0.008$ \\
\hline 3 & $\begin{array}{l}\text { S. pneumoniae } \\
\text { No.3 }\end{array}$ & Susceptible & 1 & 0.13 & 0.13 & 0.03 & 0.03 & 0.015 & 0.13 & 0.03 & 0.06 & 0.015 & $\leqslant 0.008$ & 0.03 & 0.015 & $\leqslant 0.008$ & $\leqslant 0.008$ & $\leqslant 0.008$ \\
\hline 4 & $\begin{array}{l}\text { S. pneumoniae } \\
\text { No. } 4\end{array}$ & erm B methylase (c) & $>128$ & $>128$ & 128 & 8 & 8 & 0.5 & 128 & 8 & 8 & 4 & 0.5 & 0.03 & 0.5 & 0.13 & 0.03 & 0.5 \\
\hline 5 & $\begin{array}{l}\text { S. pneumoniae } \\
\text { No. } 5\end{array}$ & erm B methylase (c) & $>128$ & $>128$ & NT & NT & NT & 0.25 & NT & 2 & 1 & 2 & 0.5 & 0.03 & 0.25 & 0.06 & 0.015 & 0.03 \\
\hline 6 & $\begin{array}{l}\text { S. pneumoniae } \\
\text { No. } 6\end{array}$ & erm B methylase (c) & $>128$ & $>128$ & 128 & 64 & 8 & 0.25 & 128 & 8 & 2 & 4 & 1 & 0.06 & 0.5 & 0.13 & 0.03 & 1 \\
\hline 7 & $\begin{array}{l}\text { S. pneumoniae } \\
\text { No. } 7\end{array}$ & erm B methylase (i) & $>128$ & 128 & 128 & 16 & 8 & 0.25 & 128 & 4 & 2 & 1 & 0.25 & 0.015 & 0.03 & 0.06 & 0.015 & 0.03 \\
\hline 8 & $\begin{array}{l}\text { S. pneumoniae } \\
\text { No. } 8\end{array}$ & erm B methylase (i) & $>128$ & 128 & 128 & 16 & 8 & 0.25 & 128 & 4 & 1 & NT & 0.25 & 0.015 & NT & 0.03 & 0.015 & 0.03 \\
\hline 9 & $\begin{array}{l}\text { S. pneumoniae } \\
\text { No. } 9\end{array}$ & mef $E$ efflux & 1 & 0.13 & 0.13 & 0.03 & 0.06 & $\leqslant 0.008$ & 0.13 & 0.03 & 0.03 & $\leqslant 0.008$ & $\leqslant 0.008$ & 0.015 & $\leqslant 0.008$ & $\leqslant 0.008$ & $\leqslant 0.008$ & 0.06 \\
\hline 10 & $\begin{array}{l}\text { S. pneumoniae } \\
\text { No. } 10\end{array}$ & mef $E$ efflux & 1 & 0.13 & 0.13 & 0.03 & 0.06 & 0.015 & 0.13 & 0.03 & 0.03 & NT & $\leqslant 0.008$ & 0.015 & NT & $\leqslant 0.008$ & $\leqslant 0.008$ & 0.06 \\
\hline 11 & $\begin{array}{l}\text { Streptococcus } \\
\text { pyogenes Cook }\end{array}$ & Susceptible & 0.13 & 0.13 & 0.06 & 0.03 & 0.06 & 0.03 & 0.13 & 0.03 & 0.06 & 0.015 & $\leqslant 0.008$ & 0.03 & $\leqslant 0.008$ & $\leqslant 0.008$ & $\leqslant 0.008$ & $\leqslant 0.008$ \\
\hline 12 & S. pyogenes No.2 & erm B methylase (c) & $>128$ & $>128$ & 128 & 16 & 2 & 0.25 & 128 & 4 & 4 & NT & 0.5 & 0.06 & 0.25 & 0.06 & 0.03 & 16 \\
\hline 13 & S. pyogenes No.3 & mef $E$ efflux & 0.25 & 0.13 & 0.25 & 0.03 & 0.06 & 0.015 & 0.13 & 0.03 & 0.06 & 0.015 & 0.015 & 0.06 & 0.015 & $\leqslant 0.008$ & $\leqslant 0.008$ & 0.25 \\
\hline 14 & $\begin{array}{l}\text { Haemophilus } \\
\text { influenzae No.1 }\end{array}$ & Susceptible & 8 & 8 & 16 & 4 & 16 & 2 & 16 & 32 & 4 & 2 & 4 & 2 & 1 & 2 & 1 & 0.5 \\
\hline 15 & H. influenzae No.2 & Susceptible & 16 & 8 & 16 & 4 & 8 & 2 & 8 & 16 & 4 & 2 & 2 & 2 & 1 & 1 & 1 & 2 \\
\hline 16 & H. influenzae No.3 & Susceptible & 16 & 32 & $>128$ & 16 & 64 & 2 & 64 & 32 & 8 & 4 & 8 & 2 & 2 & 2 & 1 & 1 \\
\hline
\end{tabular}

Abbreviations: c, constitutive; i, inducible; NT, not tested; TEL, telithromycin.

aAll anticacterial evaluations were performed as hydrochloride.

Table 2 Representative ${ }^{1} \mathrm{H}$ NMR data and MASS data of each analog. ${ }^{a}$

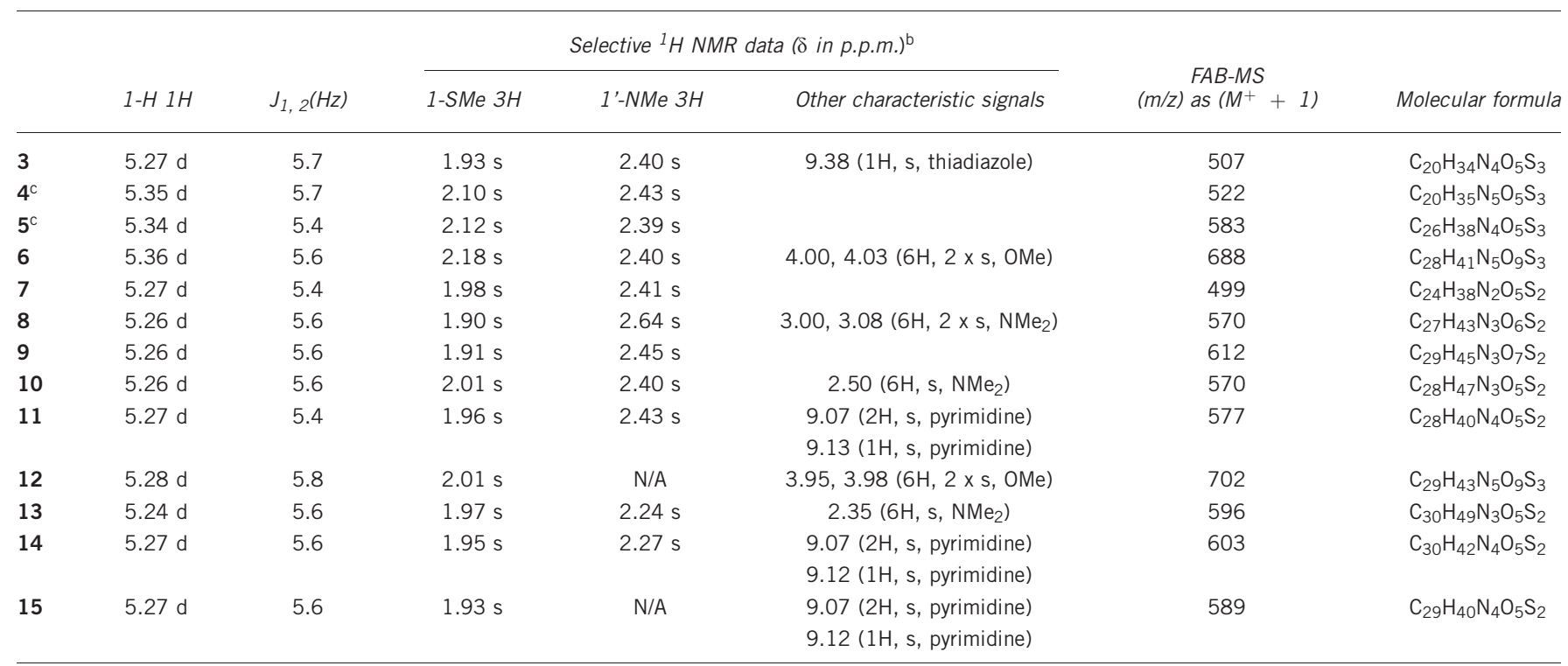

${ }^{\text {aAll }}{ }^{1} \mathrm{H}$ NMR spectra and FAB-MS were measured as free base.

$\mathrm{b}_{400 \mathrm{MHz}}$ in $\mathrm{CD}_{3} \mathrm{OD}$.

c300 $\mathrm{MHz}$ in $\mathrm{CD}_{3} \mathrm{OD}$. 
cultured on blood agar plate for S. pneumoniae, S. pyogenes and $H$. influenzae. A $5 \mu \mathrm{l}$ portion of cell suspension of the test strains having about $10^{6} \mathrm{CFU}$ per $\mathrm{ml}$ was inoculated into sensitivity disk agar (SDA, Nissui Pharmaceutical) supplemented with 5\% horse blood and incubated at $37^{\circ} \mathrm{C}$ for $20 \mathrm{~h}$. Then, minimum inhibitory concentration was measured.

\section{CONFLICT OF INTEREST}

The authors declare no conflict of interest.

\section{ACKNOWLEDGEMENTS}

The authors wish to greatly thank Professor Emeritus Masatoshi Konno for his excellent supervision.

1 Argoudelis, A. D., Coats, J. H., Mason, D. J. \& Sebek, O. K. Microbial transformation of antibiotics. III Conversion of clindamycin to 1'-demethylclindamycin and clindamycin sulfoxide by Streptomyces species. J. Antibiot. 22, 309-314 (1969)

2 Magerlein, B. J. \& Kagan, F. Lincomycin. VIII. 4'-Alkyl-1'-demethyl-4'-depropylclindamycins, Potent antibacterial and antimalarial agents. J. Med. Chem. 12, 780-784 (1969).

3 Magerlein, B. J. Lincomycin. 14. An improved synthesis and resolution of the antimalarial agent, 1'-Demethyl-4'-depropyl-4'(R)- and -(S)-pentylclindamycin hydrochloride (U-24,729A). J. Med. Chem. 15, 1255-1259 (1972).

4 Lewis, J. G. et al. Novel antimicrobial 7-methyl lincosamides: Pipecolamide analogs 44th Interscience Conference on Antimicrobial Agents and Chemotherapy, Washington DC, Poster F-1389, 2004).
5 Lewis, J., Patel, D. V., Kumar, A. S. \& Gordeev, M. F. (Vicuron Pharmaceuticals, Inc.), Preparation of lincomycin thio glycoside derivatives possessing antibacterial activity, WO 2004/016632 A2, Feb. 26 (2004).

6 Sztaricskai, F., Dinya, Z., Puskaa, M. M., Batta, G., Masuma, R. \& Ōmura, S. Semisynthetic modification of antibiotic lincomycin. J. Antibiot. 49, 941-943 (1996).

7 Schlünzen, F. et al. Structural basis for the interaction of antibiotics with the peptidy transferase centre in eubacteria. Nature 413, 814-821 (2001).

8 Bannister, B. Modification of lincomycin involving the carbohydrate portion. Part 1. The 2-O-methyl and 2-deoxy-analogues. J. Chem. Soc., Parkin Trans. I 3025-3030 (1972).

9 Houtman, R. L. (The Upjohn Company), Trimethylsilyl ether of lincomycin and its compounds, US 3,418,414, Dec. 24 (1968).

10 Ahonkhai, V. I., Cherubin, C. E., Shulman, M. A., Jhagroo, M. \& Bancroft, U. In vitro activity of U-57930E, a new clindamycin analog, against aerobic Gram-positive bacteria. Antimicrob. Agents Chemother. 21, 902-905 (1982).

11 Birkenmeyer, R. D., Kroll, S. J., Lewis, C., Stern, K. F. \& Zurenko, G. E. Synthesis and antimicrobial activity of clindamycin analogues: Pirlimycin, a potent antibacterial agent. J. Med. Chem. 27, 216-223 (1984).

12 Umemura, E. et al. (Meiji Seika Kaisha, Ltd.), Lincomycin derivative and antimicrobial agent containing the same as active ingredient. WO 2007/066805 A1, Jun. 14 (2007).

13 Wakiyama, Y. et al. (Meiji Seika Kaisha Ltd.), Lincomycin derivatives and antimicrobial agents composing the same as active ingredient. WO 2008146917 A1, Dec. 4 (2008).

14 Umemura, E. et al. (Meiji Seika Kaisha, Ltd.), Lincomycin derivatives and antimicrobial agents composing the same as active ingredient. WO 2008/146919 A1, Dec. 4 (2008)

15 Lewis, J. G., Patel, D. V., Anandan, S. K. \& Gordeev, M. F. (Vicuron Pharmaceuticals, Inc.) Lincomycin derivatives possessing antibacterial activity, US 2004/0230046 A1, Nov. 18 (2004).

16 Chen, T. et al. Novel 4'-cycloalkyl pipecolamide lincosamide analogs (45th Interscience Conference on Antimicrobial Agents and Chemotherapy, Washington DC Poster F-2036, 2005). 